\title{
Control Problem with Incomplete Information
}

\author{
Alexander N. Kvitko*, Timothey S. Taran ${ }^{\dagger}$, and Oksana S. Firyulina ${ }^{\ddagger}$ \\ Saint-Petersburg State University, 7/9 Universitetskaya nab., St. Petersburg, 199034, Russia \\ Email: *alkvit46@mail.ru, ${ }^{\dagger}$ tim9539@yandex.ru, ${ }^{\ddagger}$ firyulina.oxana@mail.ru
}

\begin{abstract}
By constructing a Luenberger-type asymptotic observer, a method of finding the control function, that ensures the translation of a class of nonlinear stationary control systems of ordinary differential equations from the initial state to a given final state taking into account the actual measured values, was developed. A constructive criterion guaranteeing the existence of solution of this problem was found. An algorithm is proposed for constructing a control function that transfer wide class of nonlinear systems of ordinary differential equations from an initial state to an fixed state. The algorithm is convenient for numerical implementation. A constructive condition is obtained for which this transfer is possible.
\end{abstract}

\section{INTRODUCTION}

One of the most important and challenging aspects of mathematical control theory is related to development of methods for constructing control functions in which different types of solutions of systems of ordinary differential equations join given points in the phase space. In many applications the control law cannot be reconstructed by the information about the complete phase state, because it is only a certain function of phase coordinates that can be measured. In this context, the problem arises of finding the required control functions from the data that has actually been measured. One approach to the solution of this problem consists in constructing in constructing an asymptotic observer. The foundations of the theory of asymptotic observes for linear-stationary systems were laid by Luenberger in his monograph [1]. In the subsequent decades this theory was generalized and extended to linear nonstationary, bilinear, and nonlinear systems of special form [2]-[7]. However, the problem of the construction of asymptotic observer for nonlinear control systems of the general form is poorly studied and is far from being solved. The situation with the problem of syntheses of terminal control of nonlinear systems is much the same [7], [8]. This paper proposes a method for solving a terminal control problem for a nonlinear control stationary system. Let us consider the controlled system of ordinary differential equations

$$
\dot{x}=f(x, u),
$$

where $x=\left(x^{1}, \ldots, x^{n}\right)^{T}, x \in R^{n}, u=\left(u^{1}, \ldots, u^{r}\right)^{T}$, $u \in R^{r}, r \leq n, t \in[0, \infty)$,

$$
\begin{gathered}
f=C^{2}\left(R^{n} \times R^{r} ; R^{n}\right), f=\left(f^{1}, \ldots, f^{n}\right)^{T}, \\
f(0,0)=0, \\
\operatorname{rank}\left(B, A B, \ldots, A^{n-1} B\right)=n, \\
A=\frac{\partial f(0,0)}{\partial x}, B=\frac{\partial f(0,0)}{\partial u},
\end{gathered}
$$

$$
\begin{gathered}
f^{i}\left(0,0, \ldots, 0, x^{n-r+1}, \ldots, x^{n}, u\right)=0, i=1, \ldots, n-r, \\
\forall x^{n-r+1} \in R^{1}, \ldots, \forall x^{n} \in R^{1}, \forall u \in R^{r}, \\
\operatorname{det}\left\{\frac{\partial f^{i}(0,0)}{\partial u^{j}}\right\} \neq 0, i=n-r+1, \ldots, n, j=1, \ldots, r .
\end{gathered}
$$

Assume that for any $t \in[0, \infty)$ one can measure the vector, $y(t) \in R^{m}, m \leq n$ which is related to the phase vector $x$ by equation

$$
y(t)=Q x(t), \operatorname{rank}(Q)=m, m<n,
$$

where $Q$ is constant matrix:

$$
\operatorname{rank}\left(Q^{T}, A^{T} Q^{T}, \ldots, A^{T n-1} Q^{T}\right)=n .
$$

From conditions (2), (3) and (6) follows the existence of $\varepsilon_{1}>0$ such that $\forall \bar{x} \in R^{r}$

$$
\|\bar{x}\|<\varepsilon_{1}
$$

equation

$$
\bar{f}(0, \ldots, 0, \bar{x}, u)=0, \bar{f}=\left(f^{n-r+1}, \ldots, f^{n}\right)^{T}
$$

defines a differentiable function $u=u(\bar{x})$ in domain (9), that satisfies (10) and the condition

$$
u(0)=0 .
$$

Let $\Gamma$ to be the $r$-dimensional plane determined by the equation $x^{i}=0, i=1, \ldots, n-r$.

Problem. Using the observes of $y(t), t \in[0, \infty)$ determined by (7), find functions $x(t) \in C^{1}[0, \infty)$ and $u(t) \in C^{1}[0, \infty)$ satisfying the system (1) and conditions

$$
x(0)=0, x(t) \rightarrow x_{1} \text { as } t \rightarrow \infty, x_{1} \in \Gamma .
$$

In (12) $x_{1}=\left(x_{1}^{1}, \ldots, x_{1}^{n}\right)^{T}, x_{1} \in \Gamma$ is a fixed vector. Let us call this pair of functions a solution of problem (1), (12).

\section{SOLUTION OF PROBLEM}

Theorem 1: Suppose conditions (2)-(7) and (8) are satisfied. Then exists $\varepsilon>0$ such that a solution of the problem (1), (2) exists

$$
\forall \bar{x}_{1}:\left\|\bar{x}_{1}\right\|<\varepsilon, \bar{x}_{1}=\left(0, \ldots, 0, \bar{x}_{1}^{n-r-1}, \ldots, \bar{x}_{1}^{n}\right)^{T} \in \Gamma,
$$

which can be obtain after solving the problem of stabilization of a linear stationary system and subsequent solution of a Cauchy problem for an auxiliary system of ordinary differential equations. 
Proof: By having fixed vector $\bar{x}_{1} \in \Gamma$ one can find $u_{1} \in R^{r}$ such, that

$$
u_{1}=u\left(\bar{x}_{1}\right), u_{1}=\left(u_{1}^{1}, \ldots, u_{1}^{r}\right)^{T} .
$$

The pair $x_{1}, u_{1}$ is satisfying the equation (10). We seek the solution of the considered problem (1), (12) in the form

$$
\begin{gathered}
x(t)=a(t)+\bar{x}_{1}, a=\left(a^{1}, \ldots, a^{n}\right)^{T}, \\
u(t)=b(t)+u_{1}, b=\left(b^{1}, \ldots, b^{r}\right)^{T} .
\end{gathered}
$$

After the substitution of (14), (15) into (1) and (7) using (2), (5), (10), (13), (14) we obtain the system and the equation, which can be represented as

$$
\begin{aligned}
& \dot{a}=P a+T b+R(a, b), \\
& P=\left\{p_{j}^{i}\right\}, p_{j}^{i}=\frac{\partial f^{i}}{\partial x^{j}}\left(\bar{x}_{1}, u_{1}\right), i, j=1, \ldots n \\
& T=\left\{t_{k}^{i}\right\}, t_{k}^{i}=\frac{\partial f^{i}}{\partial u^{k}}\left(\bar{x}_{1}, u_{1}\right), \\
& i=1, \ldots, n ; k=1, \ldots, r ; R=\left(R^{1}, \ldots, R^{n}\right)^{T}, \\
& R^{i}(a, b)=\frac{1}{2} \sum_{j=1}^{n} \sum_{s=1}^{n} \frac{\partial^{2} f^{i}}{\partial x^{j} x^{s}}(\tilde{a}, \tilde{b}) a^{j} a^{s}+ \\
& \frac{1}{2} \sum_{l=1}^{r} \sum_{k=1}^{r} \frac{\partial^{2} f^{i}}{\partial u^{l} \partial u^{k}}(\tilde{a}, \tilde{b}) b^{l} b^{k}+\frac{1}{2} \sum_{j=1}^{n} \sum_{k=1}^{r} \frac{\partial^{2} f^{i}}{\partial x^{j} \partial u^{k}}(\tilde{a}, \tilde{b}) a^{j} b^{k}, \\
& \tilde{a}=\bar{x}_{1}+\theta_{i} a, \tilde{b}=u_{1}+\theta_{i} u ; \theta_{i} \in(0,1), i=1, \ldots, n, \\
& \bar{y}(t)=Q a(t), \bar{y}(t)=y(t)-Q x_{1} .
\end{aligned}
$$

It follows from equations (27), (23)

Using the observes (19) we shall find functions $a(t), b(t)$ are satisfying system (17) and conditions

$$
a(0)=-\bar{x}_{1}, a(t) \rightarrow 0, t \rightarrow \infty .
$$

Let us choice any matrix $W$ of size $n-m \times n$ such, that

$$
\operatorname{rank}(L)=n, L=(Q, W)^{T} .
$$

Let $E$ and $D$ be the blocks of the inverse matrix $L^{-1}, L^{-1}=$ $(E, D)$, where $E$ is matrix of size $n \times m$ and $D$ is matrix of size $n \times n-m$. It is clear, that $E Q+D W=I$, where $I$ is identity matrix of size $n \times n$. Let us consider vector $\alpha(t) \in R^{n-l}$ is connecting with $a(t)$ by relation

$$
a(t)=L^{-1}(\bar{y}(t) \alpha(t))^{T}=E \bar{y}(t)+D \alpha(t) .
$$

Let us note that $(\bar{y}(t), \alpha(t))^{T}=L a(t)$. Only the vector $\alpha(t)$ in the right side of (22) needs to be estimated. By using conditions (17), (21) we obtain, that function $\alpha(t)=W a(t)$ satisfies equation

$$
\dot{\alpha}(t)=W(P D \alpha(t)+P E \bar{y}(t)+T b(t))+W R(a, b) .
$$

Initial data $\alpha_{0}$ can be chosen arbitrarily. Lets denote the estimation of the vector $\alpha(t)$ as $\hat{\alpha}(t)$. We can choose it

$$
\begin{aligned}
\dot{\delta}= & (W-V Q) P D \delta+W(R(\hat{a}, b) \\
& -R(a, b))+V Q(R(a, b)-R(\hat{a}, b)) .
\end{aligned}
$$

Let us consider the system

$$
\dot{a}=P a+T b .
$$

(considering (23) and the expression for $\hat{y}(t)$ ) to satisfy the quation

$$
\begin{aligned}
& \dot{\hat{\alpha}}(t)=W P(D \hat{\alpha}(t)+E \bar{y}(t))+V[\dot{y}(t) \\
& -Q P(D \hat{\alpha}(t)+E \bar{y}(t))]+(W-V Q) T b(t) \\
& +W R(\hat{a}, b)-V Q R(\hat{a}, b) \text {, } \\
& \hat{\alpha}(0)=\hat{\alpha_{0}}, \\
& \hat{a}(t)=E \bar{y}(t)+D \hat{\alpha}(t) \text {. }
\end{aligned}
$$

In (24) $V$ is a constant matrix to be defined. It follows from (24), (22), (19), (17) that

$$
\begin{aligned}
\dot{\hat{\alpha}}(t)= & W P(D \hat{\alpha}(t)+E \bar{y}(t)) \\
& +V Q[\dot{a}(t)-P(D \hat{\alpha}(t)+E \bar{y}(t)-T b)] \\
& +W T b(t)+W R(\hat{a}, b)-V Q R(\hat{a}, b) \\
= & W P(D \hat{\alpha}(t)+E \bar{y}(t))+V Q P(a-E \bar{y} \\
& +W T b(t)+V Q(R(a, b)-R(\hat{a}, b)) \\
& +W R(\hat{a}, b)=W P(D \hat{\alpha}(t)+E \bar{y}(t)) \\
& +V Q P D(\alpha-\hat{\alpha})+W T b(t) \\
& +V Q(R(a, b)-R(\hat{a}, b))+W R(\hat{a}, b) .
\end{aligned}
$$

Conditions (2), (4), (11) and (14) provide the existence of $\varepsilon_{2}: 0<\varepsilon_{2}<\varepsilon_{1}$ such that $\forall \bar{x}_{1}:\left\|\bar{x}_{1}\right\|<\varepsilon_{2}$

$$
\operatorname{rank}\left(T, P T, P^{2} T, \ldots, P^{n-1} T\right)=n .
$$

By using condition (31) and the well-known algorithm [7], one can find control $b(t)$ of kind

$$
b=M a
$$

to provide exponential stability of the system (30), closed by control (32)

$$
\dot{a}=(P+T M) a .
$$

Let us consider the system

$$
\dot{\delta}=(W-V Q) P D \delta .
$$

One can readily see that conditions (2), (8) and (11) implies the existence of an $\varepsilon_{3}: 0<\varepsilon_{3}<\varepsilon_{2}$ such that $\forall x_{1}:\left\|x_{1}\right\|<\varepsilon_{3}$

$$
\operatorname{rank}\left(Q^{T}, P^{T} Q^{T}, \ldots, P^{T n-1} Q^{T}\right)=n .
$$

By using condition (14), (35) and well-known algorithm [8], one can find matrix $V$ to be provide exponential stability of the system (34). Substituting the control

$$
b=M \hat{a}, \hat{a}=a+D \delta
$$


into the right-hand side of the systems (17), (29) and taking into account (36), (26) we obtain the system

$$
\begin{aligned}
\dot{a}= & (P+T M) a+T M D \delta+R(a, M(a+D \delta)), \\
\dot{\delta}= & (W-V Q) P D \delta+W((R(a+D \delta, M(a+D \delta)) \\
& -R(a, M(a+D \delta)))-V Q((R(a, M(a+D \delta)) \\
& -R(a+D \delta, M(a+D \delta))) .
\end{aligned}
$$

System (38) can be represented as

$$
\dot{\delta}=(W-V Q) P D \delta+R_{1}(a, \delta),
$$

where

$$
\begin{aligned}
R_{1}(a, \delta)= & W((R(a+D \delta, M(a+D \delta)) \\
& -R(a, M(a+D \delta)))-V Q((R(a, M(a+D \delta)) \\
& -R(a+D \delta, M(a+D \delta))) .
\end{aligned}
$$

Let

$$
\begin{gathered}
\xi=(a, \delta)^{T}, \Gamma_{1}=\left(I^{1}, O_{1}\right)_{2 n-l \times 2 n-l}, \\
\Gamma_{2}=\left(O_{2}, I^{2}\right)_{2 n-l \times 2 n-l},
\end{gathered}
$$

where $I_{n \times n}^{1}, I_{n-l \times n-l}^{2}$ are identity matrices, $O_{i}, i=1,2$ are zero matrices. By helping this denotes the systems (37), (39) can be written as

$$
\dot{\xi}=\bar{P} \xi+\bar{R}(\xi),
$$

where

$$
\bar{P}=\left(\begin{array}{cc}
P+T M & T M D \\
O_{3} & (W-V Q) P D
\end{array}\right)_{2 n-l-l \times 2 n-l},
$$

$\mathrm{O}_{3}$ is zero matrix,

$$
\bar{R}(\xi)=\left(R\left(\Gamma_{1} \xi, \Gamma_{2} \xi\right), R_{1}\left(\Gamma_{1} \xi, \Gamma_{2} \xi\right)\right)^{T} .
$$

It is follow from (20), (25) that initial data of system (41) have kind

$$
\begin{gathered}
\xi(0)=\xi_{0}=(a(0), \delta(0))^{T}=\left(-\bar{x}_{1}, \delta_{0}\right)^{T}, \\
\delta_{0}=\left(\delta_{0}^{1}, \ldots, \delta_{0}^{n-m}\right)^{T} .
\end{gathered}
$$

Initial data (42) can be chosen arbitrarily. Combining (2) and (18) we arrive at the conclusion, that in

$$
\|\xi\|<C
$$

there exist constant $L>0$. Such that

$$
\|\bar{R}(\xi)\| \leq L\|\xi\|^{2}, L>0 .
$$

By virtue of exponential stability of system

$$
\dot{\xi}=\bar{P} \xi
$$

there exists a positive definite form $V(\xi)$ such that

$$
\left.\frac{d V}{d t}\right|_{(45)} V(\xi)=-\|\xi\|^{2} .
$$

From (46) is follows that the derivative of $V(\xi)$ by virtue system (41) can be written as

$$
\left.\frac{d V}{d t}\right|_{(41)} V(\xi)=-\|\xi\|^{2}+(\operatorname{grad} V, \bar{R}(\xi)) .
$$

Evaluating the right-hand side of (47) in domain (43) with the regard for (44) we obtain the estimate

$$
\left.\frac{d V}{d t}\right|_{(41)} V(\xi)=-\|\xi\|^{2}+L\|\xi\|^{3} .
$$

Choose a constant $C_{1}>0: 0<C_{1}<C$ such that

$$
L C_{1}<1 \text {. }
$$

Then in region

$$
\|\xi\|<C_{2} .
$$

By virtue of (48), (49) we have inequality

$$
\left.\frac{d V}{d t}\right|_{(41)} V(\xi)=-\gamma\|\xi\|^{2}, \gamma>0 .
$$

On the other hand, according [8] the function $V(\xi)$ is a quadratic form satisfying of the estimates

$$
\alpha_{1}\|\xi\|^{2} \leq V(\xi) \leq \alpha_{2}\|\xi\|^{2}, \alpha_{i}>0, i=1,2 .
$$

Constants $\alpha_{i}$ are defined by matrix of quadratic form $V(\xi)$. Combining (51) and (52) we obtain

$$
V(\xi) \leq V\left(\xi_{0}\right) e^{-\frac{\gamma}{\alpha_{2}} t}, t \in[0, \infty) .
$$

Finally conditions (52) and (53) imply the estimate

$$
\|\xi(t, 0, \xi)\| \leq\left(\alpha_{2} / \alpha_{1}\right)^{\frac{1}{2}}\left\|\xi_{0}\right\| e^{-\frac{\gamma}{2 \alpha_{2}} t}, t \in[0, \infty) .
$$

Let

$$
\left\|\xi_{0}\right\|<\left(\frac{\alpha_{2}}{\alpha_{1}}\right)^{\frac{1}{2}} C_{2} .
$$

Then inequalities (54), (55) imply that the solution of system (41) with initial data (42) stays within region (50) and satisfy condition (54). After substitution of component $a(t)$ of the solution of system (41) with initial data is satisfy inequality (55) into (32), (16), (15) we obtain solution of problem (1), (12). Constant $\varepsilon>0$, which is in right side (13), can be equal as $\varepsilon=\sqrt{\frac{\alpha_{2}}{\alpha_{1}}} C_{2}$.

\section{SOLUTION OF THE INTERORBITAL FLIGHT PROBLEM}

Let us illustrate our method in more detail by solving problem of bringing a mass point with variable mass $m(t)$ moving on circular orbit at an a constant velocity in a central field of gravitation to a given point lying in the orbit plane with use of jet engines. By [9] the system of equations in deviations with respect to the above mentioned motion along a circular orbit has the form

$$
\begin{aligned}
& \dot{x}^{1}=x^{2}, \quad \dot{x}^{2}=v_{1}\left(x^{1}, x^{4}\right)+u^{1}, \\
& \dot{x}^{3}=x^{4}, \quad \dot{x}^{4}=v_{2}\left(x^{1}, x^{2}, x^{4}\right)+v_{3}\left(x^{1}\right) u^{2},
\end{aligned}
$$

where $x^{1}=r-r_{0}, x^{2}=\dot{r}, x^{3}=\psi-\alpha_{0} t, x^{4}=\dot{\psi}-\alpha_{0}$, $u_{1}=a_{r} \frac{\dot{m}}{m}, u_{2}=a_{\psi} \frac{\dot{m}}{m}, r_{0}$ is the radius of the circular orbit, $\dot{r}$ is the radial velocity; $\psi$ is the polar angle; $\dot{\psi}$ is the velocity; $a_{r}, a_{\psi}$ are the projections of the relative velocity vector of the separating particle onto the radial and the transverse direction, respectively; $m$ and $\dot{m}$ are the mass and the mass change rate 
respectively; $\alpha_{0}$ is the angular velocity of motion along the given orbit.

$$
\begin{gathered}
v_{1}=-\frac{v}{\left(x^{1}+r_{0}\right)^{2}}+\left(x^{1}+r_{0}\right)\left(x^{4}+\alpha_{0}\right)^{2}, \\
v_{2}=-2 \frac{x^{2}\left(x^{4}+\alpha_{0}\right)}{x^{1}+r_{0}}, v_{3}=\frac{1}{x^{1}+r_{0}},
\end{gathered}
$$

where $v=v^{0} M ; v^{0}$ is the gravity constant; $M$ is the mass of Earth, $\alpha_{0}=\sqrt{v / r_{0}^{3}}, x=\left(x^{1}, \ldots, x^{4}\right)^{T}, u=\left(u^{1}, u^{2}\right)^{T}$, $\bar{x}_{1}=\left(x_{1}^{1}, 0, x_{1}^{3}, 0\right)^{T}, u_{1}=\left(u_{1}^{1}, u_{1}^{2}\right)^{T}, u_{1}^{1}=-v_{1}\left(\bar{x}_{1}\right), u_{1}^{2}=$ $-v_{2}\left(\bar{x}_{1}\right) / v_{3}\left(\bar{x}_{1}\right)$. The matrices $P$ and $T$ in the right-hand side of system (30) are

$$
\begin{gathered}
P=\left\|\begin{array}{cccc}
0 & 1 & 0 & 0 \\
a_{21} & 0 & 0 & a_{24} \\
0 & 0 & 0 & 1 \\
0 & a_{42} & 0 & 0
\end{array}\right\|, T=\left\|\begin{array}{lc}
0 & 0 \\
1 & 0 \\
0 & 0 \\
0 & v_{3}\left(\bar{x}_{1}\right)
\end{array}\right\|, \\
a_{21}=\frac{\partial v_{1}}{\partial x_{1}}\left(\bar{x}_{1}\right), a_{24}=\frac{\partial v_{1}}{\partial x_{4}}\left(\bar{x}_{1}\right), a_{42}=\frac{\partial v_{2}}{\partial x_{2}}\left(\bar{x}_{1}\right) .
\end{gathered}
$$

After the of the stabilization problem for system (30) we find matrix $M$ as:

$$
M=\left\|\begin{array}{cccc}
-\left(a_{21}+a_{24} a_{42}+11\right) & -6 & -\frac{6}{a_{42}} & 0 \\
\frac{a_{42}}{\beta} & 0 & 0 & -\frac{1}{\beta}
\end{array}\right\|,
$$

We consider the following measurement equation

$$
y=Q x, Q=\left\|\begin{array}{llll}
0 & 1 & 0 & 0 \\
0 & 0 & 1 & 0
\end{array}\right\|
$$

Let us put matrix $W$ as

$$
W=\left\|\begin{array}{llll}
0 & 0 & 0 & 1 \\
1 & 0 & 0 & 0
\end{array}\right\|
$$

Using $Q, W$ we obtain matrices $D, V$ :

$$
D=\left\|\begin{array}{llll}
0 & 0 & 0 & 1 \\
1 & 0 & 0 & 0
\end{array}\right\|^{T}, V=\left\|\begin{array}{cc}
0 & 6 \\
5 / a_{21} & -6 a_{24} / a_{21}
\end{array}\right\| .
$$

\section{NUMERICAL SIMULATION}

In the course of numerical simulation, we integrate the auxiliary system (37), (38) with initial data (42), on the interval $t \in[0,12]$, where $x_{1}^{1}=100 \mathrm{~m}, x_{1}^{i}=0, i=\overline{2,4}$, $\delta_{0}^{1}=0.00001, \delta_{0}^{2}=0.00001, r_{0}=7 \times 10^{6} \mathrm{~m}$. Matrices $P$, $T, Q, M, W, V, D$ have kind (56)-(60). The figures (Fig. 1, Fig. 2, Fig. 3) represent of the change of the functions $x^{1}(t)$, $x^{2}(t), x^{3}(t), x^{4}(t)$ and $u^{1}(t), u^{2}(t)$.

\section{REFERENCES}

[1] D. G. Luenberger, "Observers for multivariable systems," IEEE Trans. Aut. Control, vol. 18, pp. 190-197, 1966.

[2] W. M. Wonham, "Dynamic observers-Geometric theory," IEEE Trans. Aut. Control, vol. 15, no. 2, pp. 258-259, 1970.

[3] J.R.Ŕoman and T.E. Bullock, "Design of minimal order stable observes for linear functions of the state via realization theory," IEEE Trans. Aut. Control, vol. 20, pp. 613-632, 1975.

[4] Yu. S. Kan and A.I. Kibzun, "Stabilization of Dynamic System under Uncertain and Random Perturbations," Autom. Telemech., no. 12, pp. 7584, 1990.
[5] V. N. Afanasiev, Dynamic systems with incomplete information. Algorithmic synthesizing, (in Russian). Moscow, Russia: Komkniga, 2007.

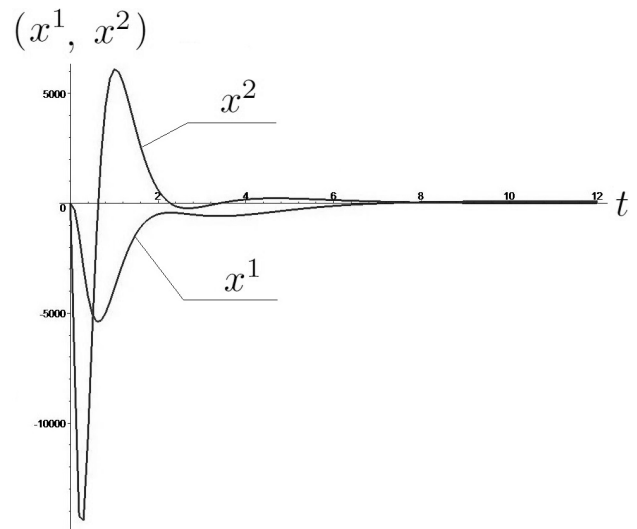

Fig. 1. The plot of the change of the phase coordinates $x^{1}(t)$ and $x^{2}(t)$.

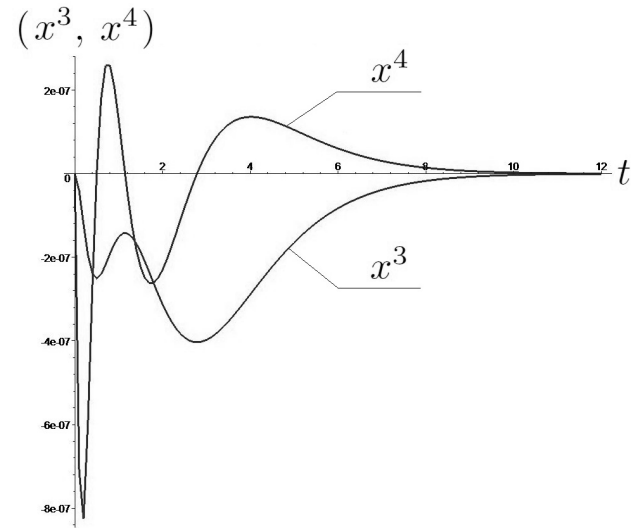

Fig. 2. The plot of the change of the phase coordinates $x^{3}(t)$ and $x^{4}(t)$.

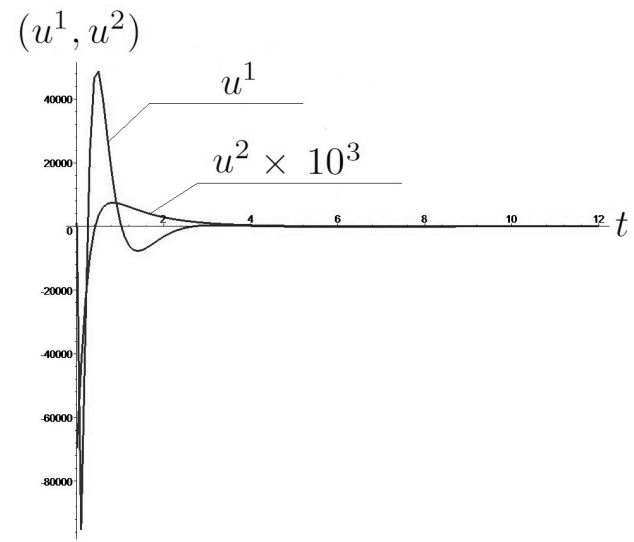

Fig. 3. The plot of the change of the control functions $u^{1}(t)$ and $u^{2}(t)$.

[6] A. N. Kvitko and D. B. Yakusheva, "Synthesis of discrete stabilization for a nonlinear stationary control system under incomplete information," (in Russian), Vestnik St. Petersburg Univ. Math., vol. 20, no. 2, pp. 65-72, 2012.

[7] I.E.Zuber, "A terminal control of nonlinear systems," (in Russian), Vestnik St. Petersburg Univ. Math., vol. 34, no. 3, pp. 12-15, 2001.

[8] V.I.Zubov, Lectures on control theory, (in Russian). Moscow, Russia: Nauka, 1975.

[9] N.N. Krasovsky, Theory of Motion Control, (in Russian). Moscow, Russia: Nauka, 1968. 